\title{
Modified Toll Gate System with Enhanced Security Using FPGA
}

\author{
D.Punniamoorthy , Mr.P.Vimal Kumar, M.E \\ P.G.Scholar Dept of Electronics \& Communication Engineering Sri Muthukumaran Institute of Technology \\ Chennai,Tamilnadu,India. \\ Assistant Professor Dept of Electronics \& Communication Engineering Sri Muthukumaran Institute of \\ Technology Chennai,Tamilnadu,India.
}

\begin{abstract}
Toll gates are usually considered an inconvenience by travelers not only for the cost of the toll, but also for the delays at toll booths, toll roads and bridges. In order to ensure a steady flow of traffic, both staff and drivers require easy access to an efficient communication system covering the specific requirements of toll gates. In this way, hitches can be resolved while maintaining a convenient toll gate system. Security systems can also be added, which will further enhance the system. In this paper we are adding various safety \& security measures to the modern toll gate system to avoid the security and safety threads. This paper is designed using a FPGA Xilinx Spartan $3 A N$ with the help of VLSI technology.

Keywords: 6LoWPAN, CoAP, Internet of Things, REST,Sensor, Sensor Network, Web of Things
\end{abstract}

\section{Introduction}

The toll gate system using RFID technology enables the electronic collection of toll payments. This technology has been studied by researchers and applied in various highways, bridges, and tunnels requiring such a process. This system is capable of determining if the car is registered or not, and then informing the authorities of toll payment violations, debits etc. The most obvious advantage of this technology is the opportunity to eliminate congestion in tollbooths, especially during festive seasons when traffic tends to be heavier than normal. It is also a method by which to curb complaints from motorists regarding the inconveniences involved in manually making payments at the tollbooths. Other than this obvious advantage is that it could also benefit the toll operators.

The benefits for the motorists through this system can be fewer or shorter queues at toll plazas, faster and more efficient service (no exchanging toll fees by hand), the ability to make payments by keeping a balance on the card itself or by loading a registered credit card. The use of postpaid toll statements (no need to request for receipts). Other general advantages for the motorists include fuel savings and reduced mobile emissions by reducing or eliminating deceleration, waiting time, and acceleration.

Meanwhile, for the toll operators, the benefits through this system are lowered toll collection costs, better audit control by centralized user accounts, expanded capacity without building more infrastructures.

This system commonly utilizes radio frequency identification (RFID) technology. RFID is a generic term used to identify technologies utilizing radio waves to automatically identify people or objects. RFID technology was first introduced in 1948 when Harry Stockman wrote a paper exploring RFID technology entitled, "Communication by Means of Reflected Power". RFID technology has evolved since then and has been implemented in various applications such as in warehouse management, library system, attendance system, theft prevention and so on. In general, RFID is used for tracking, tracing, and identifying objects.

\section{Existing System Problems}

The existing system consists of a microcontroller, RFID reader, RFID Tag, stepper motor, and bill printer. The reader retrieves the information about the ID number and identifies the vehicle. Then for the tax to be collected the bill is printed at the time of exit. The stepper motor here is used to open and close the gate automatically.

In the existing system, though there is an RFID reader the tax collection is manual and not automated. There are no security features such as identifying a stolen vehicle etc. The toll tax which is collected for all the vehicles is the same and the tax collected is not based on the load carried by the vehicle.

\section{Proposed System}

The proposed system makes sure that the traffic at the toll gates is streamlined and security is also present. The tax which is collected is based on the load carried by the vehicle. Through this system we can also identify stolen vehicles. 


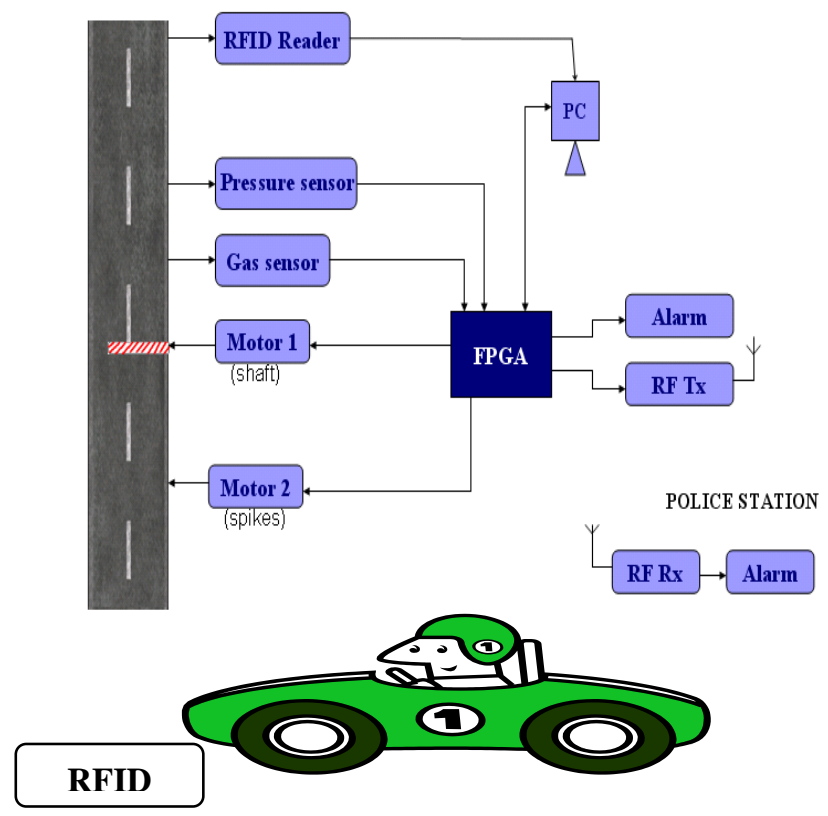

Figure 1 Block Diagram of proposed system

\section{BLOCK DIAGRAM DESCRIPTION}

Figure 1 shows the proposed system. An RFID tag is installed on each vehicle with read/write memory. An RFID reader at the gate reads this data from the vehicle as it approaches the toll booth. RFID reader communicates with PC using UART (Universal Asynchronous Receiver/Transmitter). UART takes bytes of data and transmits the individual bits in a sequential fashion. PC consists of the entire database which is updated periodically. Now after reading the information, PC compares the data in the database and allows the access accordingly by opening/closing the gate. A time stamp and the entry count are also written onto the RFID tag each time the vehicle passes through the gate. This data is used to print a daily or monthly bill for toll collection from the vehicles. This way even stolen vehicles can be identified.

The pressure of the vehicle is obtained using the pressure sensor and accordingly the pressure of the vehicle is displayed on the display. A counter is used to count the number of vehicles. The amount on the basis of weight $\&$ the count of vehicles is also displayed on the screen. The amount to be paid is automatically deduced from the respective bank account.

If a vehicle carries any kind of gas that shouldn't be carried, the gas sensor detects the gas in the vehicle. In case if there is any kind of gas that is detected, the RF transmitter is used to alert the nearby police station and an alarm is enabled to alert the surrounding areas. Subsequently motor 1 is used to close the gate and simultaneously motor 2 is used to pull up the spikes in order to puncture the vehicle.

Radio frequency identification (RFID) technology relates to short-range wireless communications and uses the radio frequency to read certain information on a device known as a tag shown in figure 3.4. They are commonly used for wireless data communication with $\mathrm{R} / \mathrm{W}$ devices at distances ranging from a few millimeters to several meters. Due to its flexibility and convenience RFID technology is now used in a variety of applications including supply chain processing, security systems, inventory control, mail delivery and counterfeit product prevention.

There are three fundamental RFID architectures in use today: passive, battery assisted passive (BAP), and active. Passive RFID tags do not carry their own energy source. They operate by harvesting energy from the reader and send data by reflecting energy back to the reader. Active RFID operates by utilizing energy from a battery or an equivalent local energy source. They send data to a reader by producing a low-power modulated signal. BAP RFID is a hybrid architecture that sends data by reflecting energy from the reader in the same manner as passive RFID, but utilizes a battery for its overall operation.

Most RFID tags contain integrated circuit (IC) chip, to store and process data, and also an integrated antenna, which is used as the communication interface with read and write antenna system. The IC chip requires power to operate, which in the initial designs was supplied by a battery. Recently, most applications require the tags to be small and inexpensive, so the so-called passive (no-battery) chips became common and widely used. 


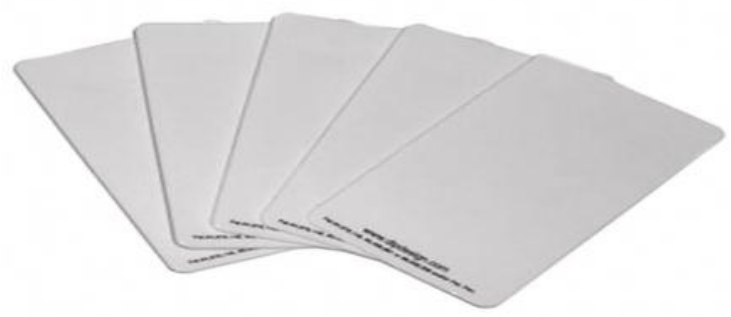

Figure 3 RFID Tag

Here we use a passive RFID tag as shown in figure 3.3. Passive RFID tags are the most physically robust RFID tags. A passive tag is an RFID tag does not contain a battery and the power is supplied by the reader. Such tags can have memory anywhere from 64 bits to $1 \mathrm{~KB}$.

When an RFID reader has to read data in the tag, radio waves from the reader are encountered by an RFID tag, the coiled antenna within the tag forms a magnetic field. The tag draws power from it, energizing the circuits in the tag. The tag then retrieves and sends the information encoded in the tag's memory.

The majority of passive tags use EEPROM memory. Some are laser programmed at the silicon level. The tag functions without a battery and these have a useful life of twenty years or more.

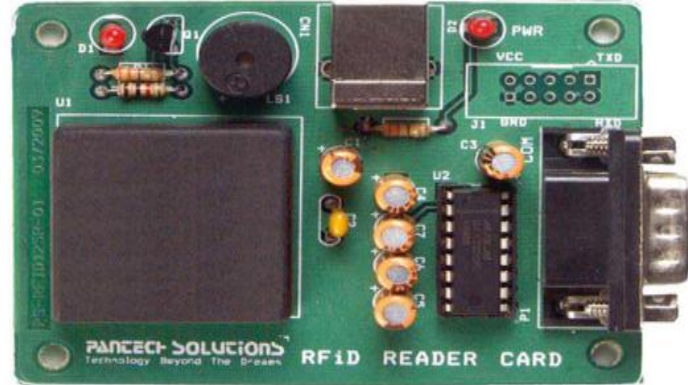

Figure 4 RFID Reader

In our project RFID reader as shown in figure 4 is placed at the gate. It reads the data and compares it with the data in the database in the PC and allows the access accordingly by opening/closing the gate.

\section{FPGA (FIELD-PROGRAMMABLE GATE ARRAY)}

The FPGA is an integrated circuit that contains many (64 to over 10,000) identical logic cells that can be viewed as standard components. Each logic cell can independently take on any one of a limited set of personalities. The individual cells are interconnected by a matrix of wires and programmable switches. A user's design is implemented by specifying the simple logic function for each cell and selectively closing the switches in the interconnect matrix. The array of logic cells and interconnects form a fabric of basic building blocks for logic circuits. Complex designs are created by combining these basic blocks to create the desired circuit. Field Programmable means that the FPGA's function is defined by a user's program rather than by the manufacturer of the device. A typical integrated circuit performs a particular function defined at the time of manufacture. In contrast, the FPGA's function is defined by a program written by someone other than the device manufacturer.

$$
\text { FPGA structure }
$$

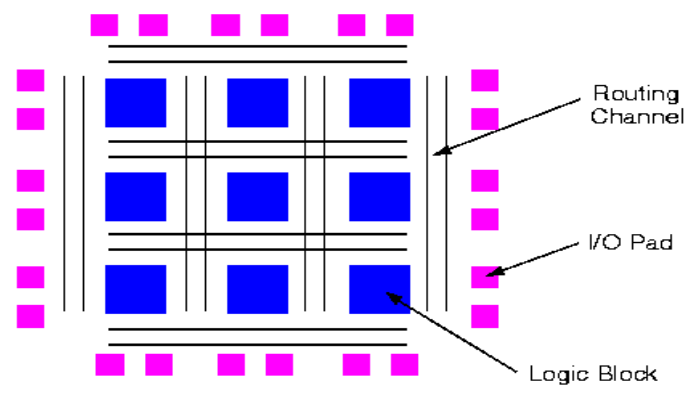

Figure 5 FPGA structure 
Figure 5 shows the FPGA structure. The logic cell architecture varies between different device families. Generally speaking, each logic cell combines a few binary inputs (typically between 3 and 10) to one or two outputs according to a boolean logic function specified in the user program. In most families, the user also has the option of registering the combinatorial output of the cell, so that clocked logic can be easily implemented. The cell's combinatorial logic may be physically implemented as a small look-up table memory (LUT) or as a set of multiplexers and gates. LUT devices tend to be a bit more flexible and provide more inputs per cell than multiplexer cells at the expense of propagation delay.

\section{FPGAS VS MICROCONTROLLERS}

Microcontrollers are based on CPU architecture. As all CPUs, they execute instructions in a sequential manner. FPGAs are programmable logic and run in a parallel fashion.

Microcontrollers have on-chip peripherals that also execute in parallel with their CPU. But they are still much less configurable than programmable logic.

\section{Pressure Sensor}

A piezoelectric sensor as shown in figure 6 is a device that uses the piezoelectric effect to measure pressure, acceleration, strain or force by converting them to an electrical charge. Here a simple pressure sensor is used to protect door or window. It generates a loud beep when somebody tries to break the door or window. The alarm stops automatically after three minutes. The circuit uses a piezo element as the pressure sensor.

Piezo buzzer exploits the piezoelectric property of the piezo electric crystals. The piezoelectric effect may be direct piezoelectric effect in which the electric charge develops as a result of the mechanical stressor reverse or indirect piezoelectric effect (Converse piezoelectric effect) in which a mechanical force such as pressure develops due to the application of an electric field.

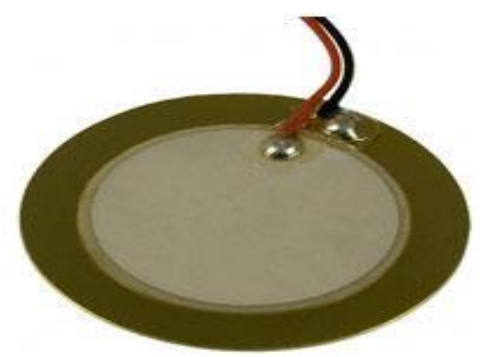

Figure 6 Piezo electric sensor

A typical example of direct piezoelectric effect is the generation of measurable amount of piezoelectricity when the Lead Zirconate Titanate crystals are deformed by mechanical or heat stress. The Lead Zirconate Titanate crystals also shows indirect piezoelectric effect by showing pressure when an electric potential is applied.

\section{OPERATION}

Operation of pressure sensor is very simple. Here we have two plates that is one is input plate and the other is output plate, whenever pressure is applied as shown in figure 7 then these two plates come into contact we get voltage as a output then this output is send to the FPGA in turn it shows the weight of the vehicles is shown in the display as per our project. Accordingly toll tax is calculated. It is made up of a piezoelectric crystal. Depending on how a piezoelectric material is cut, three main modes of operation can be distinguished into transverse, longitudinal, and shear.

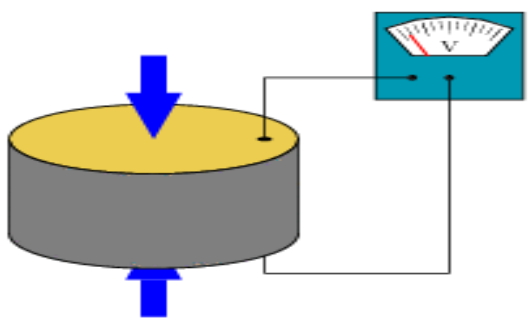

Figure 8 Pressure sensor operation 
LM 324

LM324 is a 14pin IC consisting of four independent operational amplifiers (op-amps) compensated in a single package. Op-amps are high gain electronic voltage amplifier with differential input and usually a singleEnded output. The output voltage is many times higher than the voltage difference between input terminals of an op-amp. These op-amps are operated by a single power supply and thus the need for a dual supply is eliminated. They can be used as amplifiers, comparators, oscillators, rectifiers etc. The figure 9 shows the pin diagram and pin details of LM 324.

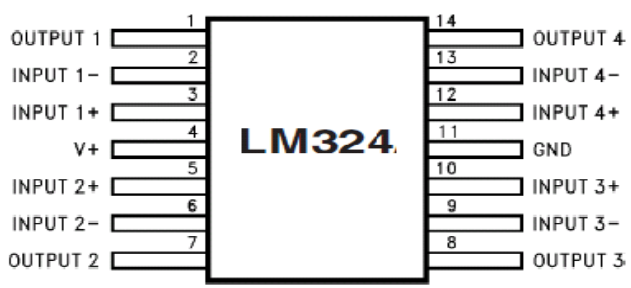

Figure 9 pin diagram and pin details of LM 324

\section{GAS SENSOR}

The Flammable Gas and Smoke sensors can detect the presence of combustible gas and smoke at concentrations from 300 to $10,000 \mathrm{ppm}$. Owing to its simple analog voltage interface, the sensor requires one analog input pin from the FPGA.
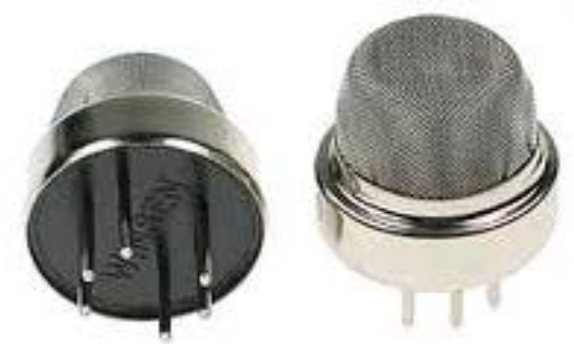

Figure 10 Gas Sensor (type-MQ2)

The product can detect the pressure of the smoke and send the output in the form of analog signals. Our range can function at temperature ranging from -20 to 50 degree Celsius and consume less than $150 \mathrm{~mA}$ at $5 \mathrm{~V}$.

Sensitive material of MQ-2 gas sensor in the figure 10 is $\mathrm{SnO} 2$ (Tin dioxide), which with lower conductivity in clean air. When the target combustible gas exist, the sensor's conductivity is higher along with the gas concentration rising.

MQ-2 gas sensor has high sensitivity to LPG, Propane and Hydrogen, also could be used to Methane.

\section{Structure And Configuration}

Structure and configuration of MQ-2 gas sensor is shown as figure 11, sensor composed by micro AL2O3 ceramic tube, Tin Dioxide ( $\mathrm{SnO} 2)$ sensitive layer, measuring electrode and heater are fixed into a crust made by plastic and stainless steel net. The heater provides necessary work conditions for work of sensitive components. The enveloped MQ-2 has 6 pin, four of them are used to fetch signals, and other two are used for providing heating current.
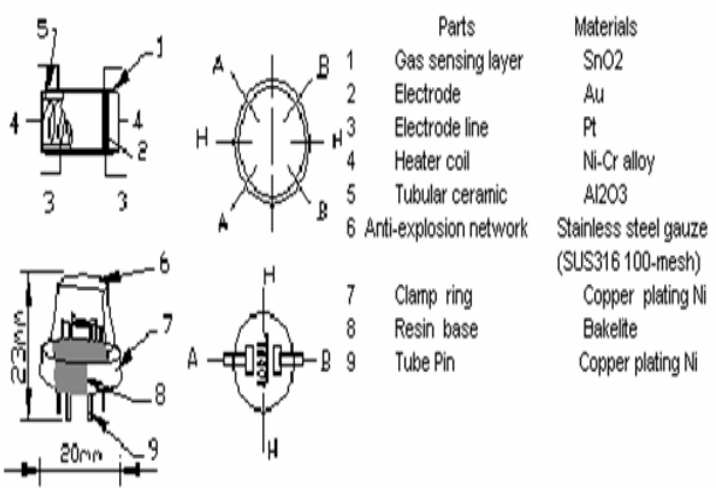

Copper plating N

Figure 11 Structure and Configuration of MQ2 sensor 


\section{LM 358}

LM 358 series consists of two independent, high gain, internally frequency compensated operational amplifiers which were designed specifically to operate from a single power supply over a wide range of voltages. Operation from split power supplies is also possible and the lower power supply current drain is independent of the magnitude of the power supply voltage. The figure $\mathbf{1 2}$ shows the pin diagram and pin details of LM 358.

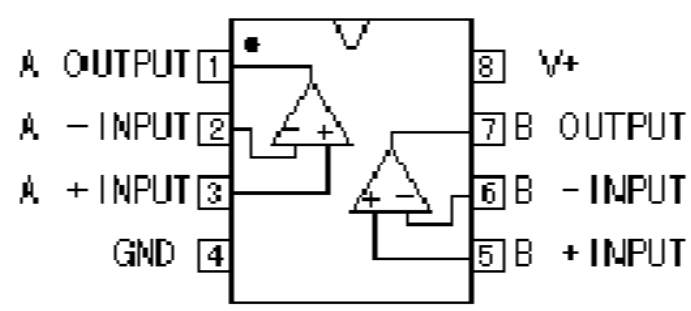

Figure 12 Pin details of LM 358

\section{WIRELESS COMMUNICATION MODULE}

A general RF communication block diagram is shown in figure 13. Since most of the encoders/decoders/microcontrollers are TTL compatible and mostly inputs by the user will be given in TTL logic level. Thus, this TTL input is to be converted into serial data input using an encoder or a microcontroller. This serial data can be directly read using the RF Transmitter, which then performs ASK (in some cases FSK) modulation on it and transmit the data through the antenna. In the receiver side, the RF Receiver receives the modulated signal through the antenna, performs all kinds of processing, filtering, demodulation, etc and gives out a serial data. This serial data is then converted to a TTL level logic data, which is the same data that the user has input.

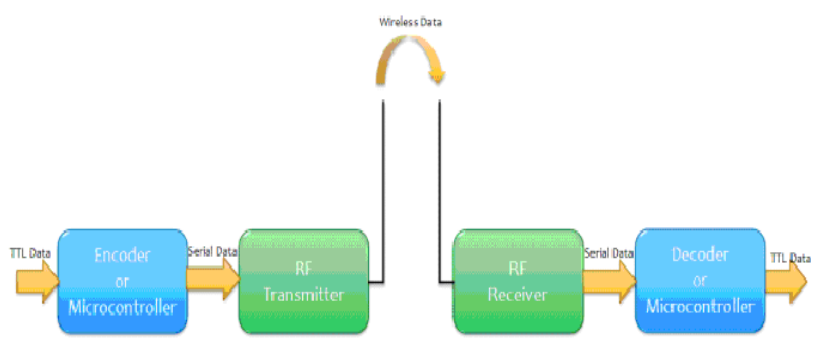

Figure 13 RF communication block diagram

\section{RF TRANSMITTER}

An RF transmitter generates radio frequency waves in its circuits, and to the carrier signal, it adds the information part by modulating the carrier signal. This composite signal (carrier plus information) is then fed to an antenna.

The antenna induces a corresponding signal into the atmosphere, by altering the Electric and Magnetic fields at the same frequency. The following are its features.

- Range in open space(Standard Conditions) : 100 Meters

- TX Frequency Range : $433.92 \mathrm{MHz}$

- TX Supply Voltage : 3V $\sim 6 \mathrm{~V}$

- TX Out Put Power : 4 12 Dbm

- Low Power Consumption

- Easy For Application

The pin diagram of RF transmitter is shown in figure. 


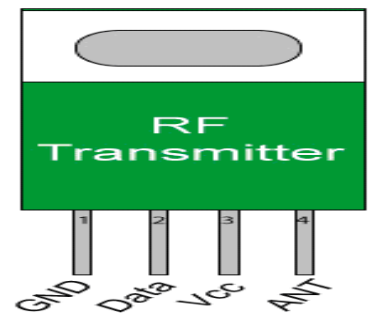

Figure 14 Pin diagram of RF transmitter

\section{RF RECEIVER}

An RF receiver receives the signal from the atmosphere, from its own antenna. The receiver antenna is often quite simple and the signal level is typically of a few microvolts. This gets rid of unwanted signals and amplifies only the wanted ones. The receiver circuits then strip the information part of the signal from the carrier part. The following are its features

- Range in open space(Standard Conditions) : 100 Meters

- RX Receiver Frequency : $433 \mathrm{MHz}$

- RX Typical Sensitivity : 105 Dbm

- RX Supply Current : $3.5 \mathrm{~mA}$

- RX IF Frequency : $1 \mathrm{MHz}$

- Low Power Consumption

- Easy For Application

- RX Operating Voltage : 5V

The pin diagram of RF receiver is shown in figure 15 .

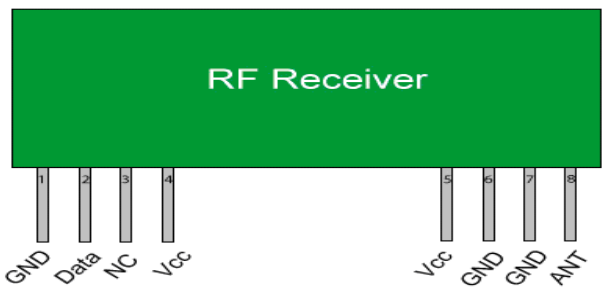

Figure 3.15 pin diagram of RF receiver

\section{MOTOR}

The steel can forms the body of the motor in addition to an axle, a nylon end cap and two battery leads. If the battery leads of the motor are hooked up to a flashlight battery, the axle will spin. If leads are reversed, it will spin in the opposite direction. Here are two other views of the same motor. The nylon end cap is held in place by two tabs that are part of the steel can. By bending the tabs back, the end cap can be made free and remove it. Inside the end cap are the motor's brushes. These brushes transfer power from the battery to the commutator as the motor spins. The figure 16 shows all the parts of the DC motor.

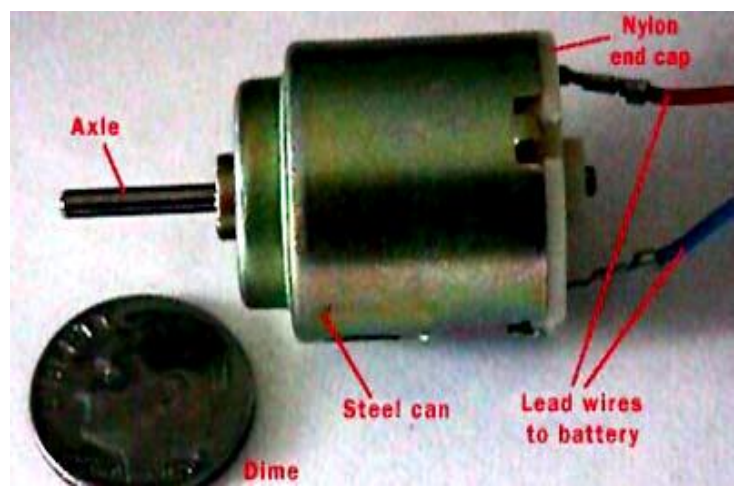

Figure 16 parts of a DC motor 


\section{RELAY WITH ULN2003A}

A relay is an electrical hardware device having an input and output gate. The output gate consists in one or more electrical contacts that switch when the input gate is electrically excited. A relay is an electrical switch that opens and closes under the control of another electrical circuit. In the original form, the switch is operated by an electromagnet to open or close one or many sets of contacts.

\section{RELAY OPERATION}

Relay uses an electromagnet. This is a device consisting of a coil of wire wrapped around an iron core. When electricity is applied to the coil of wire becomes magnetized, hence the term is called electromagnet. The A B and C terminals are a SPDT switch which is controlled by the electromagnet. When electricity is applied to V1 and V2, the electromagnet acts upon the SPDT switch so that the B and C terminals are connected. When the electricity is disconnected, then the $\mathrm{A}$ and $\mathrm{C}$ terminals are connected. It is important to note that the electromagnet is magnetically linked to the switch but the two are NOT linked electrically. The figure 17 shows the relay used here.

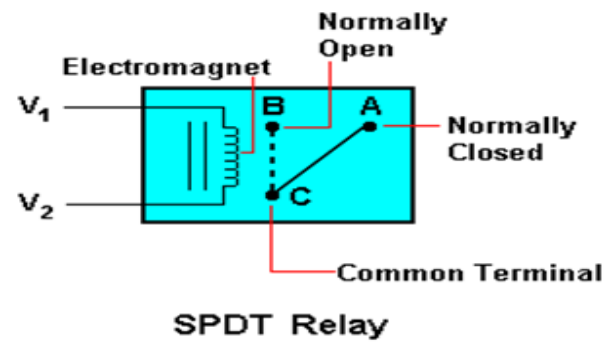

Figure 17 Relay

General operation of relay is when a current flows through the coil, the resulting magnetic field that attracts an armature that is mechanically linked to a moving contact. The movement either makes or breaks a connection with a fixed contact. When the current to the coil is switched off, the armature is returned by a force, approximately half as strong as the magnetic force, to its relaxed position. Usually this is a spring, but gravity is also used commonly in industrial motor starters. Most relays are manufactured to operate quickly.

If the coil is energized with direct current, a diode is often placed across the coil to dissipate the energy from the collapsing magnetic field at deactivation, which would otherwise generate a voltage spike dangerous to semiconductor circuit components. If the coil is designed to be energized with alternating current (AC), a small copper "shading ring" can be crimped to the end of the solenoid, creating a small out-of-phase current which increases the minimum pull on the armature during the AC cycle.

\section{Prototype Results}

The results obtained using our proposed system is as follows
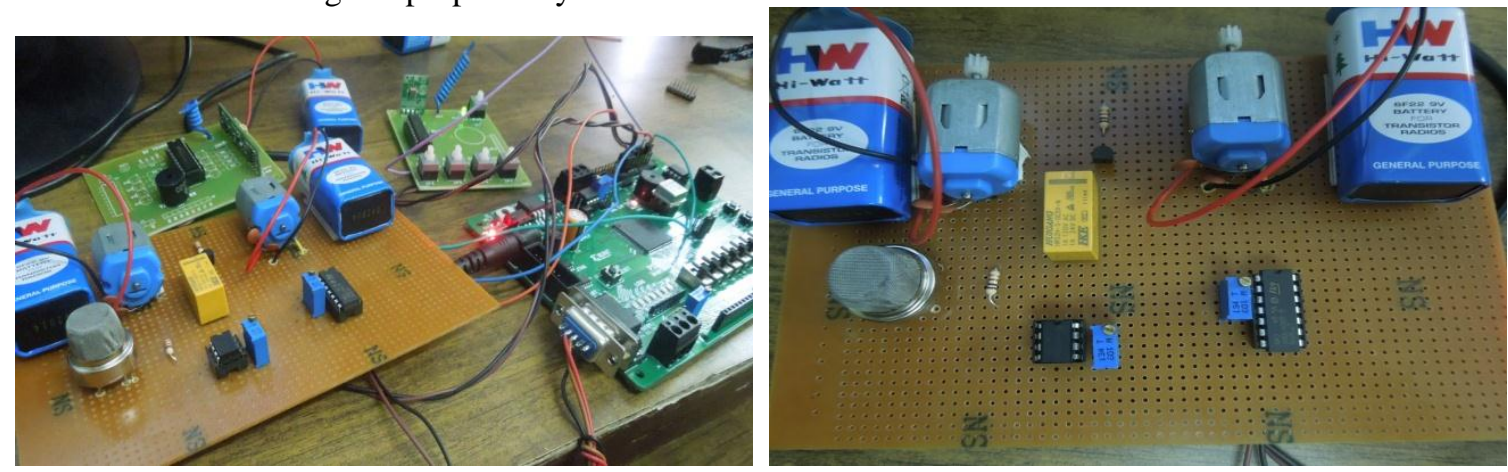

Picture 1 \& 2: Overall Hardware

In the above figure consist of a FPGA, RF transmitter, RF receiver, RFID Reader, gas sensor, pressure sensor, motors and relay which are connected together. Our project is a unmanned toll gate system which makes toll tax to be collected automatically, provides security, and traffic free which does not require any manual supervision. If any vehicle is stolen are gas is carried then it immediately alarms the surroundings and to the nearby police station in the mean while it switches on the motor 1 and 2 . Here motor 1 is a shaft and motor 2 produces spikes. 

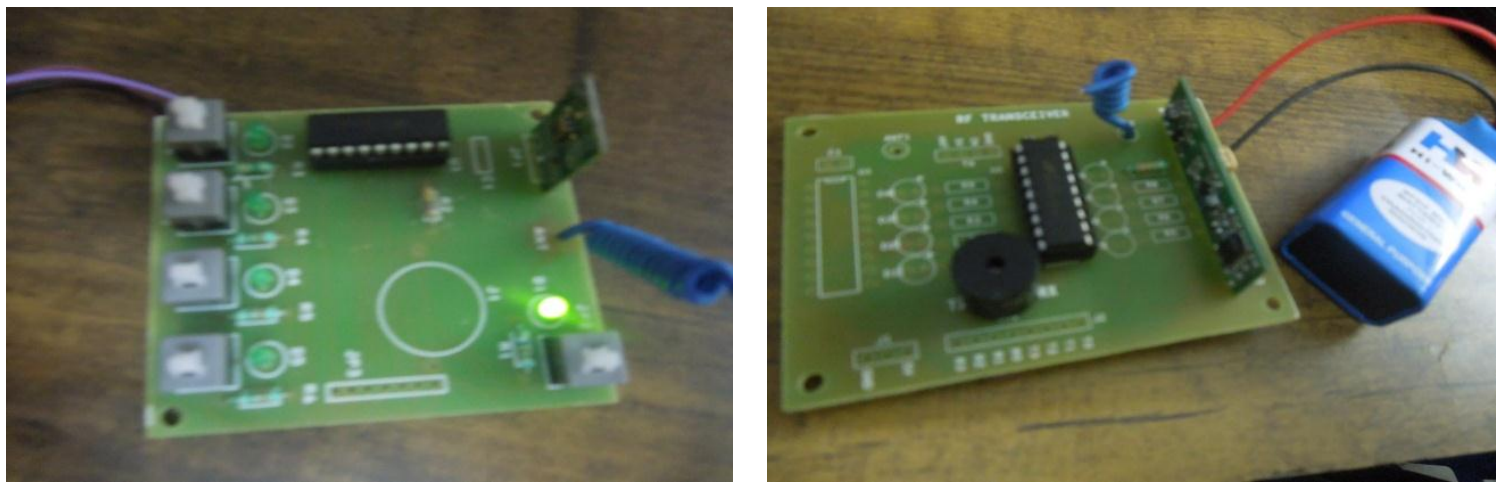

Picture 3 \& 4: RF Transmitter \& receiver

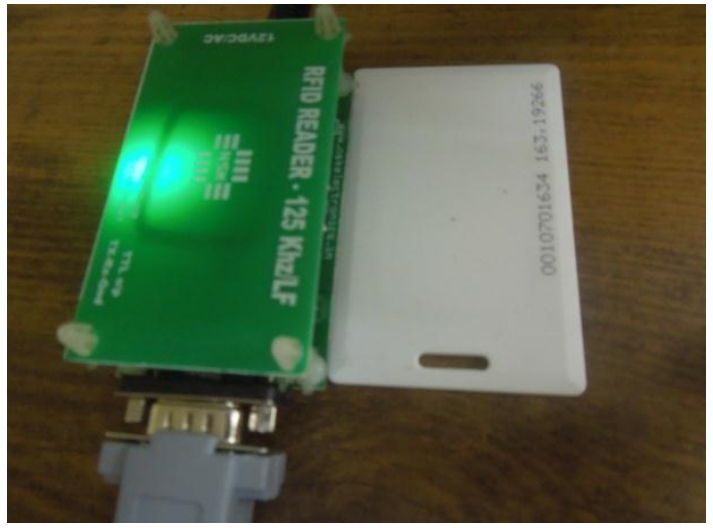

Picture 5: RFID Reader and RFID Tag

The above figure shows the motor and gas module, RF transmitter, RF receiver, RFID reader RFID Tag. These modules work simultaneously which is controlled by FPGA. Here RFID reader uses to read the information from the RFID Tag which is installed in all the vehicles. The information is compared with the data in PC database. If the vehicle is found to be stolen then it alters the surroundings and through the RF transmitter it signals the RF receiver which is in the nearby police station. Similarly the same procedure is carried out when a gas is detected by a gas sensor.

Pressure sensor is used to detect the pressure of the vehicle and accordingly the toll tax is detected from their account automatically. The program is loaded into the FPGA first using Xilinx software. Then using visual basic we implement in system. Visual basic window is opened first. The port numbers are entered based on the com ports that are connected.

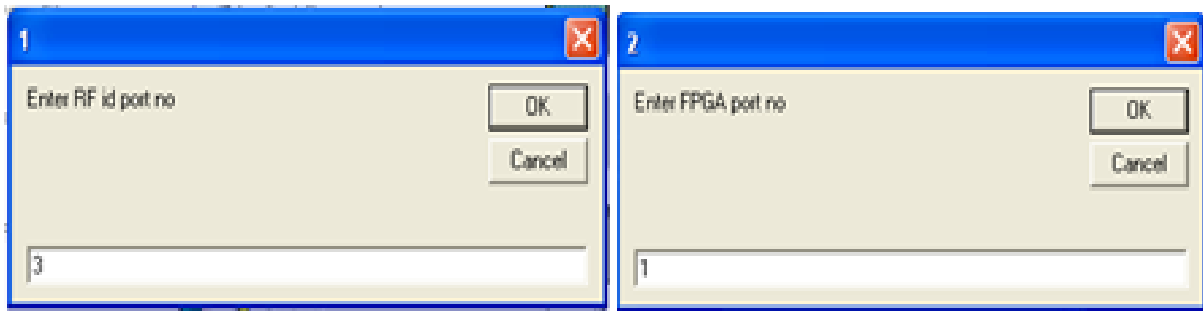

Picture 7 \& 8: Once the port numbers are given, the RFID tag is matched with the reader.

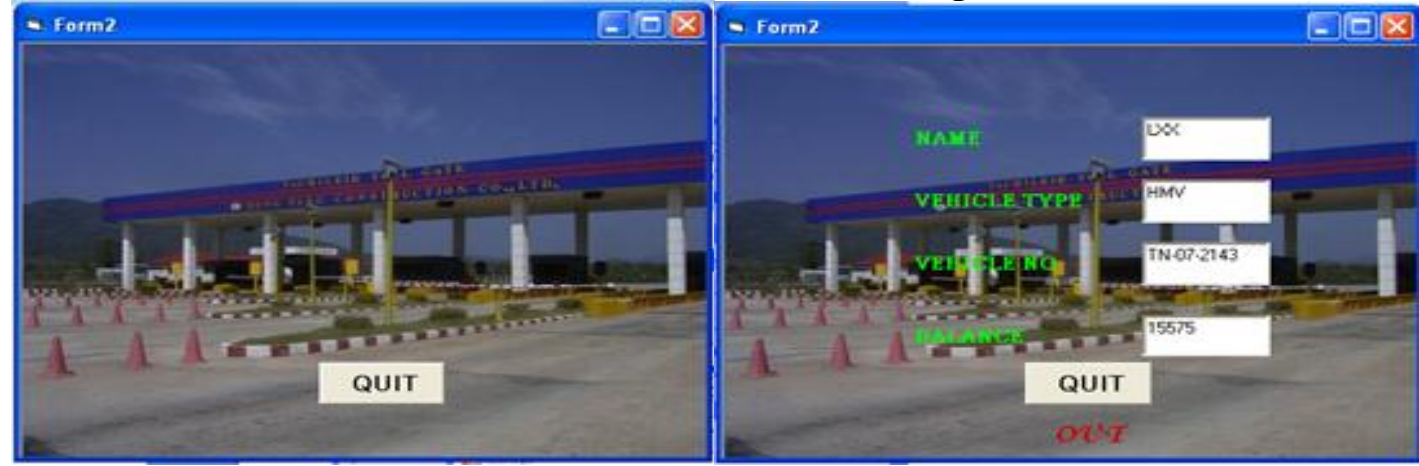

Picture 9 \& 10: Layout \& Amount and pressure chart 
After the reader identifies the tag the vehicle details are displayed. When the pressure is given the balance amount is deducted.

\section{Conclusion}

Our system is a user friendly toll fee method which can save time and reduce traffic congestion at toll gates and provide solution for users to reach their destination without wastage of time. It gives the toll authorities the flexibility to set variable pricing for toll services and thus a fair policy of tax collection can be followed. This way there is no loss incurred by a person carrying a vacant vehicle. With the hike of fuel prices in mind, consumption of fuel is also considered here as the deceleration, acceleration and idling is completely eliminated. Here there is no cash transaction for the toll lanes, so cash handling is reduced. Thus difficulties with cash handling are eliminated and this way aid in enhanced audit control by centralizing user accounts. Information such as vehicle count over the time of the day, date, time etc can be obtained due to the deployment of this technology. This helps in making decisions regarding the pricing strategies for the toll providers. It also helps planner to estimate the travel time that aid in designing decisions.

\section{References}

[1] Kin Seong Leong, Mun Leng Ng, Member, IEEE, Alfio R. Grasso, Peter H. Cole, "Synchronization of RFID Readers for Dense RFID Reader Environments", International Symposium on Applications and the Internet Workshops (SAINTW'06), 2005

[2] Manish Buhptani, Shahram Moradpour, "RFID Field Guide - Developing Radio Frequency Identification Systems", Prentice Hall, 2005, pp 7-9, 16-225, 160, 231

[3] Raj Bridgelall, Senior Member, IEEE, " Introducing a Micro-wireless Architecture for Business Activity Sensing ", IEEE International Conference RFID, April 16-17,2008

[4] Sewon Oh, Joosang Park, Yongioon Lee, "RFID-based Middleware System for Automatic Identification", IEEE International Conference on Service Operations and Logistics, and Information, 2005

[5] Shi-Cho Cha Kuan-Ju Huang Hsiang-Meng Chang, " An Efficient and Flexible Way to Protect Privacy in RFID Environment with Licenses ", IEEE International Conference RFID, April 16-17,2008

[6] Urachada Ketprom, Chaichana Mitrpant, Putchapan Lowjun, "Closing Digital Gap on RFID Usage for Better Farm Management", PICMET 2007, 5-9 August 07 